\title{
NEW ROTATIONAL INTEGRALS IN SPACE FORMS, WITH AN APPLICATION TO SURFACE AREA ESTIMATION
}

\author{
Ximo Gual-Arnau, Castelló de la Plana, Luis M. Cruz-Orive, Santander
}

(Received April 29, 2015)

\section{Cordially dedicated to Professor Vratislav Horálek on his 90th birthday, and to the memory of Professor Ivan Saxl}

Abstract. A surface area estimator for three-dimensional convex sets, based on the invariator principle of local stereology, has recently motivated its generalization by means of new rotational Crofton-type formulae using Morse theory. We follow a different route to obtain related formulae which are more manageable and valid for submanifolds in constant curvature spaces. As an application, we obtain a simplified version of the mentioned surface area estimator for non-convex sets of smooth boundary.

Keywords: critical point; height function; submanifold in space forms; invariator principle; local stereology; rotational formulae; surface area estimation

MSC 2010: 53C65

\section{INTRODUCTION}

In recent years, new rotational integral formulae have been developed ([1], [7], [8]). This was motivated by the invariator principle of stereology ([5], see also [14], p. 285). In these formulae, integration over all $r$-planes is replaced with invariant integration of a measurement function over all $r$-planes through a fixed point. In particular, in [5], a convenient method was proposed to estimate the surface area of a convex set from an isotropic plane section (called a pivotal section) through a fixed point in terms of the support function of the pivotal section. Recently, in [15], the pertinent formula ([5], equation (3.2)) has been generalized to non convex

Work was supported by the UJI project P11B2012-24 and the PROMETEOII/2014/062 project. 
sets, and new rotational Crofton formulae have thereby been developed using Morse theory. Further, in [16], the mentioned new generalization has been adapted to estimate the surface area in three-dimensional microscopy. In the present paper, we follow a different route to obtain related generalizations which are more manageable and valid for submanifolds in space forms of constant curvature. In particular, we arrive at a surface area representation which constitutes a simplification of the analogous formula given in [15], [16]. The subsequent estimation procedure is thereby simplified - the basic task consists in identifying the local maxima and minima of the height function of the pivotal section.

\section{A RELATION BETWEEN DENSities of totally GEODESiC SUbManifoldS} IN SPACE FORMS

Let $M_{\lambda}^{n}$ denote a simply connected Riemannian manifold of sectional curvature $\lambda$ (namely, the sphere for $\lambda>0$, the hyperbolic space for $\lambda<0$ and the Euclidean space $\mathbb{R}^{n}$ for $\left.\lambda=0\right)$. Further, let $L_{r}^{n}$ denote an $r$-plane $(r \leqslant n)$, namely a totally geodesic submanifold of dimension $r$ in $M_{\lambda}^{n}$, and let $\mathrm{d} L_{r}^{n}$ denote the corresponding density, invariant under the group of Euclidean and non-Euclidean motions.

Let $M^{q}$ be a compact differentiable manifold of dimension $q$ embedded in $M_{\lambda}^{n}$. Santalo's formula shows that the volume of $M^{q}$ can be expressed in terms of the integral of the volume of the intersection of $M^{q}$ with all the planes $L_{r}^{n}$, assuming that $r+q \geqslant n$ ([13], equation (14.69)). While the latter formula was obtained for the Euclidean case, in [13], p. 309 (Section 4 of Chapter 17), Santaló shows that the same formula extends to $M_{\lambda}^{n}$ without any change.

An $r$-plane through a fixed point $O$ in $M_{\lambda}^{n}$, and its invariant density, are denoted by $L_{r[0]}^{n}$ and $\mathrm{d} L_{r[0]}^{n}$, respectively $([13],[12])$. One of the first problems considered in integral geometry was to determine the densities $\mathrm{d} L_{r}^{n}$ and $\mathrm{d} L_{r[0]}^{n}$. For lines in $\mathbb{R}^{2}$ the invariant density $\mathrm{d} L_{1}^{2}$ was proposed by Crofton (see [4]), whereas the invariant densities for lines and planes in $M_{\lambda}^{3}$ were obtained by Cartan [3] in 1896. In 1935, Blaschke [2] introduced the density $\mathrm{d} L_{r}^{n}$ for $\mathbb{R}^{n}$. Following Blaschke's methodology, Petkantschin [11] and Santaló [13] obtained the corresponding densities for $\lambda>0$ and $\lambda<0$, respectively.

In this section, we generalize the expressions obtained in [7] for the density of $r$-planes in $M_{\lambda}^{n}$ in terms of the density $\mathrm{d} L_{p[0]}^{n}$ of $p$-planes through a fixed point $O$, of the density $\mathrm{d} L_{r}^{p}$ of $r$-planes in $L_{p[0]}^{n}$, and of the distance $\varrho$ from $O$ to $L_{r}^{p}$. For the Euclidean case, the result can be deduced from equation (49) in [11]. From the new expression, new rotational versions of Santaló's formula, and equivalences among them, are obtained in Section 3. 
Henceforth, the following notation will be used:

$$
s_{\lambda}(\varrho)=\left\{\begin{array}{ll}
\lambda^{-1 / 2} \sin (\varrho \sqrt{\lambda}), & \lambda>0, \\
\varrho, & \lambda=0, \\
|\lambda|^{-1 / 2} \sinh (\varrho \sqrt{|\lambda|}), & \lambda<0,
\end{array} \quad c_{\lambda}(\varrho)= \begin{cases}\cos (\varrho \sqrt{\lambda}), & \lambda>0, \\
1, & \lambda=0, \\
\cosh (\varrho \sqrt{|\lambda|}), & \lambda<0 .\end{cases}\right.
$$

The density $\mathrm{d} L_{r}^{n}$ of $r$-planes in $M_{\lambda}^{n}$ can be expressed in terms of the distance $\varrho$ from a fixed point $O$ to $L_{r}^{n}$, of the density $\mathrm{d} L_{r+1[0]}^{n}$ of an $(r+1)$-plane through $O$ containing $L_{r}^{n}$, and of the density $\mathrm{d} L_{r}^{r+1}$ for $r$-planes contained in $L_{r+1[0]}^{n}$, as follows ([7], Corollary 3.1):

$$
\mathrm{d} L_{r}^{n}=s_{\lambda}^{n-r-1}(\varrho) \mathrm{d} L_{r}^{r+1} \mathrm{~d} L_{r+1[0]}^{n} .
$$

Theorem 2.1. For $r \in\{0,1, \ldots, n-2\}$ and $p \in\{r+1, r+2, \ldots, n\}$, the following relation between densities is satisfied:

$$
\mathrm{d} L_{p[r+1]}^{n} \mathrm{~d} L_{r}^{n}=s_{\lambda}^{n-p}(\varrho) \mathrm{d} L_{r}^{p} \mathrm{~d} L_{p[0]}^{n},
$$

where $\mathrm{d} L_{p[r+1]}^{n}$ denotes the density for $p$-planes in $M_{\lambda}^{n}$ that contain the fixed $(r+1)$ plane $L_{r+1[0]}^{n}$.

P r o of. As justified in [13], p. 309, before equation (17.55), from the expressions of the densities of planes in $M_{\lambda}^{n}$ it follows that some density decompositions (such as [13], equation (12.53)) have the same form regardless of the sign of $\lambda$. Therefore, multiplying both sides of equation $(2.2)$ by $\mathrm{d} L_{p[r+1]}^{n}$, and bearing equation (12.53) of [13] in mind, we obtain

$$
\mathrm{d} L_{p[r+1]}^{n} \mathrm{~d} L_{r}^{n}=s_{\lambda}^{n-p}(\varrho) s_{\lambda}^{p-r-1}(\varrho) \mathrm{d} L_{r}^{r+1} \mathrm{~d} L_{r+1[0]}^{p} \mathrm{~d} L_{p[0]}^{n} .
$$

Applying again (2.2), we get the result.

Note that (2.2) is a special case of (2.3) for $p=r+1$.

Corollary 2.2. The density $\mathrm{d} L_{r}^{n}$ satisfies the following identity:

$$
\mathrm{d} L_{r}^{n}=s_{\lambda}^{n-p}(\varrho) \mathrm{d} L_{r}^{p} \mathrm{~d} L_{p[p-r-1]}^{n},
$$

where $\mathrm{d} L_{p[p-r-1]}^{n}$ denotes the density for $p$-planes in $M_{\lambda}^{n}$ that contain a fixed plane of dimension $p-r-1$ through $O$.

Pro of. As justified in [13], p. 309, the densities given in (12.26) and (12.27) of [13] also hold in $M_{\lambda}^{n}$. Therefore,

$$
\mathrm{d} L_{p[0]}^{n}=\mathrm{d} L_{p[r+1]}^{n} \mathrm{~d} L_{p[p-r-1]}^{n},
$$

and the result follows from (2.3). 


\section{Rotational versions of SANTAló's Formula}

Let $M^{q}$ be a compact differentiable manifold of dimension $q$ embedded in $M_{\lambda}^{n}$. Assume that $r+q \geqslant n$ and consider the set of $r$-planes in $M_{\lambda}^{n}$. Santaló's formula (equation (14.69) of [13], which is valid in $M_{\lambda}^{n}$ ), states that

$$
\int_{M^{q} \cap L_{r}^{n} \neq \emptyset} \sigma_{r+q-n}\left(M^{q} \cap L_{r}^{n}\right) \mathrm{d} L_{r}^{n}=\frac{O_{n} \ldots O_{n-r} O_{r+q-n}}{O_{r} \ldots O_{0} O_{q}} \sigma_{q}\left(M^{q}\right),
$$

where $\sigma_{k}$ denotes $k$-dimensional volume, and $O_{k}=2 \pi^{(k+1) / 2} / \Gamma((k+1) / 2)$ is the surface area of the $k$-dimensional unit sphere.

In this paper, we focus on the special case $r+q=n$. Then equation (14.70) of [13], which is valid in $M_{\lambda}^{n}$, gives

$$
\int_{M^{n-r} \cap L_{r}^{n} \neq \emptyset} N\left(M^{n-r} \cap L_{r}^{n}\right) \mathrm{d} L_{r}^{n}=\frac{O_{n} \ldots O_{n-r+1}}{O_{r} \ldots O_{1}} \sigma_{n-r}\left(M^{n-r}\right),
$$

where $N$ denotes number, so that $N\left(M^{n-r} \cap L_{r}^{n}\right)$ denotes the number of points of the intersection $M^{n-r} \cap L_{r}^{n}$.

Next we apply Theorem 2.1 to obtain new rotational formulae from (3.2).

Proposition 3.1. Let $r \in\{0,1, \ldots, n-2\}$ and $p \geqslant r+1$. Also, let $M^{n-r} \subset M_{\lambda}^{n}$ be a compact submanifold of class $C^{p-r+1}$. Then

$$
\begin{aligned}
& \sigma_{n-r}\left(M^{n-r}\right) \\
& =\frac{1}{c_{n, r, p}} \int_{M^{n-r} \cap L_{p[0]}^{n} \neq \emptyset}\left[\int_{M_{p}^{n-r} \cap L_{r}^{p} \neq \emptyset} s_{\lambda}^{n-p}(\varrho) N\left(M_{p}^{n-r} \cap L_{r}^{p}\right) \mathrm{d} L_{r}^{p}\right] \mathrm{d} L_{p[0]}^{n},
\end{aligned}
$$

where $M_{p}^{n-r}=M^{n-r} \cap L_{p[0]}^{n}$, and

$$
c_{n, r, p}=\frac{O_{n} \ldots O_{n-r+1} O_{n-r-2} \ldots O_{n-p}}{O_{r} \ldots O_{1} O_{p-r-2} \ldots O_{1} O_{0}}
$$

Thus, first we fix $L_{p[0]}^{n}$ and integrate with respect to $\mathrm{d} L_{r}^{p}$ over all planes in $L_{r}^{p}$ which intersect $M_{p}^{n-r}$, and then we integrate with respect to $\mathrm{d} L_{p[0]}^{n}$ over all planes $L_{p[0]}^{n}$ which intersect $M^{n-r}$. Further, $M^{n-r}$ is assumed to be of class $C^{k}$ with $k=\max \left\{1, \operatorname{dim}\left(M_{p}^{n-r}\right)+p-n+1\right\}=p-r+1$. By virtue of Appendix A from [8], this choice ensures that $M_{p}^{n-r} \cap L_{p[0]}^{n}$, where $L_{p[0]}^{n}$ is a generic $p$-plane, is also of class $C^{k}$ (and dimension $p-r$ ). The need to specify the order $k$ of smoothness is explained in [10]. In Theorem 4.1, one has $p=r+1$, whereby $M^{n-r}$ is assumed to be of class $C^{2}$. 
Proof. As explained above, the submanifold $M_{p}^{n-r}$ is of class $C^{p-r+1}$ and dimension $p-r$, whereby $M_{p}^{n-r} \cap L_{r}^{p}$ is the set of intersection points. Now, bearing in mind equation (12.36) of [13], namely,

$$
\int_{\text {Total }} \mathrm{d} L_{p[r+1]}^{n}=\frac{O_{n-r-2} \ldots O_{n-p}}{O_{p-r-2} \ldots O_{1} O_{0}}
$$

and making use of equation (2.3) in equation (3.2), the result follows.

The next corollary shows that for the special case $p=r+1$, equation (3.3) yields equation (15) from [7] with $q=n-r$.

Corollary 3.2. Within each $p$-plane $L_{p[0]}^{n}$, consider $(r+1)$-planes $L_{r+1[0]}^{p}$. Then $\sigma_{n-r}\left(M^{n-r}\right)$ admits a rotational expression which may be obtained directly by substituting $p=r+1$ on the right-hand side of equation (3.3), namely,

$$
\sigma_{n-r}\left(M^{n-r}\right)=\frac{1}{c_{n, r}} \int_{M^{n-r} \cap L_{r+1[0]}^{n} \neq \emptyset} \alpha\left(M^{n-r} \cap L_{r+1[0]}^{n}\right) \mathrm{d} L_{r+1[0]}^{n},
$$

where

$$
\alpha\left(M^{n-r} \cap L_{r+1[0]}^{n}\right)=\int_{\mathcal{C} \cap L_{r}^{r+1} \neq \emptyset} s_{\lambda}^{n-r-1}(\varrho) N\left(C \cap L_{r}^{r+1}\right) \mathrm{d} L_{r}^{r+1},
$$

with

$$
c_{n, r}=\frac{O_{n} \ldots O_{n-r+1}}{O_{r} \ldots O_{1}}
$$

where $\mathcal{C}$ represents the curve $M_{r+1}^{n-r}=M^{n-r} \cap L_{r+1[0]}^{n}$ (of class $\left.C^{2}\right)$ in $L_{r+1[0]}^{n}$.

Proof. For $n=p$, equation (2.2) yields

$$
\mathrm{d} L_{r}^{p}=s_{\lambda}^{p-r-1}(\varrho) \mathrm{d} L_{r}^{r+1} \mathrm{~d} L_{r+1[0]}^{p} .
$$

Therefore, equation (3.3) can be written as

$$
\sigma_{n-r}\left(M^{n-r}\right)=\frac{1}{c_{n, r, p}} \int_{M^{n-r} \cap L_{p[0]}^{n} \neq \emptyset} \alpha\left(M^{n-r} \cap L_{p[0]}^{n}\right) \mathrm{d} L_{p[0]}^{n},
$$

with

$$
\begin{aligned}
& \alpha\left(M^{n-r} \cap L_{p[0]}^{n}\right) \\
& =\int_{M_{p}^{n-r} \cap L_{r+1[0]}^{p} \neq \emptyset}\left[\int_{M_{r+1}^{p-r} \cap L_{r}^{r+1} \neq \emptyset} s_{\lambda}^{n-r-1}(\varrho) N\left(C \cap L_{r}^{r+1}\right) \mathrm{d} L_{r}^{r+1}\right] \mathrm{d} L_{r+1[0]}^{p},
\end{aligned}
$$


where, by virtue of Theorem A.1 from [8], $M_{r+1}^{p-r}=M_{p}^{n-r} \cap L_{r+1[0]}^{p}$ is a curve of class $C^{2}$ in $L_{r+1[0]}^{p}$. Now, making use in equation (3.10) of the well-known identity [13]

$$
\mathrm{d} L_{r+1[0]}^{p} \mathrm{~d} L_{p[0]}^{n}=\mathrm{d} L_{p[r+1]}^{n} \mathrm{~d} L_{r+1[0]}^{n}
$$

and bearing equation (3.5) in mind, the rotational formula (3.6) is obtained.

\section{Morse type REPRESENTATION AND GEOMETRICAL INTERPRETATiONS}

In this section, a geometric interpretation of equation (3.7) is given in terms of the critical points of height functions. Of particular interest will be the special case $r=1$, whereby $M^{n-1}$ is a hypersurface, and the rotational formula is obtained by intersecting $M^{n-1}$ with a 2-plane $L_{2[0]}^{n}$.

The density $\mathrm{d} L_{r}^{r+1}$ may be decomposed as (see [13])

$$
\mathrm{d} L_{r}^{r+1}=c_{\lambda}^{r}(\varrho) \mathrm{d} \varrho \mathrm{d} u_{r}
$$

where $\mathrm{d} u_{r}$ denotes the surface area element of the $r$-dimensional unit sphere and $c_{\lambda}^{r}(\varrho)=\left(c_{\lambda}(\varrho)\right)^{r}$, where $c_{\lambda}(\varrho)$ is defined in equation (2.1). Then, for the cases $\lambda=0$ (Euclidean) and $\lambda<0$ (hyperbolic), we may write

$$
\alpha\left(M^{n-r} \cap L_{r+1[0]}^{n}\right)=\int_{\mathbb{S}^{r}} \mathrm{~d} u_{r} \int_{0}^{\infty} s_{\lambda}^{n-r-1}(\varrho) c_{\lambda}^{r}(\varrho) N\left(\mathcal{C} \cap L_{r}^{r+1}\right) \mathrm{d} \varrho,
$$

whereas, for the case $\lambda>0$ (spherical),

$$
\alpha\left(M^{n-r} \cap L_{r+1[0]}^{n}\right)=\int_{\mathbb{S}^{r}} \mathrm{~d} u_{r} \int_{0}^{\pi /(2 \sqrt{\lambda})} s_{\lambda}^{n-r-1}(\varrho) c_{\lambda}^{r}(\varrho) N\left(\mathcal{C} \cap L_{r}^{r+1}\right) \mathrm{d} \varrho
$$

where $L_{r}^{r+1}$ is the $r$-plane expressed in terms of its distance $\varrho$ from the fixed point $O$, perpendicular to the geodesic defined from the direction $u_{r}$ from $O$, and $N(\mathcal{C} \cap$ $\left.L_{r}^{r+1}\right)=0$ whenever $\mathcal{C} \cap L_{r}^{r+1}=\emptyset$.

Next we give a geometrical interpretation of $\alpha\left(M^{n-r} \cap L_{r+1[0]}^{n}\right)$, based on the critical points of height functions.

Let $u_{r}$ denote a unit vector in $\mathbb{S}^{r} \subset T_{O} L_{r+1[0]}^{n}$. The geodesic $\gamma_{u_{r}}: \mathbb{R} \rightarrow L_{r+1[0]}^{n}$ with $\gamma_{u_{r}}(0)=O$ and $\gamma^{\prime}(0)=u_{r}$ is given by $\gamma_{u_{r}}(t)=c_{\lambda}(t) O+s_{\lambda}(t) u_{r}$. Given $u_{r}$, let $h_{u_{r}}: L_{r+1[0]}^{n} \rightarrow \mathbb{R}$ be the height function whose level hypersurfaces are just the $r$-planes $L_{r}^{r+1}$ perpendicular to the geodesic $\gamma_{u_{r}}(t)$. Note that in the Euclidean case $(\lambda=0)$, this height function coincides with the standard height function considered in [15]. We suppose that the level hypersurface $L_{r}^{r+1}$ is oriented in such a way that 
the unit vector $\nu(p)$, perpendicular to the level set $L_{r}^{r+1} \subset L_{r+1[0]}^{n}$ at $p$, is given by $\nu(p)=\operatorname{grad}\left(h_{u_{r}}\right)(p) /\left\|\operatorname{grad}\left(h_{u_{r}}\right)(p)\right\|$, where $\operatorname{grad}\left(h_{u_{r}}\right)$ denotes the gradient of $h_{u_{r}}$.

We consider the height function $\left.h_{u_{r}}\right|_{\mathcal{C}}: \mathcal{C} \rightarrow \mathbb{R}$, which is generally a Morse function, and apply the Morse theory to $\left.h_{u_{r}}\right|_{\mathcal{C}}(\mathrm{cf}$. [10]).

Let $p \in \mathcal{C}$ be a critical point of $\left.h_{u_{r}}\right|_{\mathcal{C}}$ along $\mathcal{C}$. Then there is a level hypersurface $L_{r}^{r+1}$ of $h_{u_{r}}$ which satisfies $T_{p} \mathcal{C} \subset T_{p} L_{r}^{r+1}$.

We assume that $\left.h_{u_{r}}\right|_{\mathcal{C}}$ is an excellent Morse function for almost all $u_{r} \in \mathbb{S}^{r}$, which means that all of the critical points in the direction $u_{r}$ from $O$ are non-degenerate, and no two of them lie on the same level hypersurface (i.e. they have different critical values).

Note that the critical points of $\left.h_{u_{r}}\right|_{\mathcal{C}}$ and $\left.h_{\left(-u_{r}\right)}\right|_{\mathcal{C}}$ coincide, whereas the corresponding critical values are the same but with opposite signs. Because the integrals in (4.2) and (4.3) are defined for $\varrho \geqslant 0$, only nonnegative critical values are involved in the relevant integrals.

Let $p_{i} \in \mathcal{C}, i=1, \ldots, m$, be the critical points of the excellent Morse function $\left.h_{u_{r}}\right|_{\mathcal{C}}$, and

$$
0 \leqslant \varrho_{1}<\varrho_{2}<\ldots<\varrho_{m} \quad\left(\text { with } \varrho_{m} \leqslant \frac{1}{2} \pi / \sqrt{\lambda} \text { for } \lambda>0\right)
$$

the corresponding nonnegative critical values, where $\left.h_{u_{r}}\right|_{\mathcal{C}}\left(p_{i}\right)=\varrho_{i}$. To each critical point $p_{i}$ we assign an index $\varepsilon_{i}=+1$ if $p_{i}$ is a local maximum of $\left.h_{u_{r}}\right|_{\mathcal{C}}$, and an index $\varepsilon_{i}=-1$ if $p_{i}$ is a local minimum of $\left.h_{u_{r}}\right|_{\mathcal{C}}$. For $r<n \in\{1,2, \ldots\}$ define

$$
I_{n-r-1, r}(\varrho)=\int s_{\lambda}^{n-r-1}(\varrho) c_{\lambda}^{r}(\varrho) \mathrm{d} \varrho .
$$

Then for $\lambda=0$,

$$
I_{n-r-1, r}(\varrho)=\frac{\varrho^{n-r}}{n-r}
$$

For $\lambda \neq 0$ and for any given pair $(n, r)$, the integral $I_{n-r-1, r}(\varrho)$ may be evaluated explicitly with the aid of a mathematical software package such as Mathematica ${ }^{\circledR}$ or Maple ${ }^{\mathrm{TM}}$. In all cases, we set $I_{n-r-1, r}(0)=0$.

Theorem 4.1. Let $M^{n-r} \subset M_{\lambda}^{n}$ be a submanifold of class $C^{2}$. Then the intersection $M^{n-r} \cap L_{r+1[0]}^{n}$ is in general a curve $\mathcal{C}$ of class $C^{2}$ in $L_{r+1[0]}^{n}$. Further, assume that $\left.h_{u_{r}}\right|_{\mathcal{C}}$ is an excellent Morse function for almost all $u_{r} \in \mathbb{S}^{r}$. Then for $r \in\{0,1, \ldots, n-2\}$ and $\lambda \leqslant 0$,

$$
\alpha\left(M^{n-r} \cap L_{r+1[0]}^{n}\right)=2 \int_{\mathbb{S}^{r}}\left(\sum_{k=1}^{m} \varepsilon_{k} I_{n-r-1, r}\left(\varrho_{k}\right)\right) \mathrm{d} u_{r},
$$


where $m$ represents the number of critical points of the height function $\left.h_{u_{r}}\right|_{\mathcal{C}}$ corresponding to the direction $u_{r}$; and for $\lambda>0$,

$$
\begin{aligned}
& \alpha\left(M^{n-r} \cap L_{r+1[0]}^{n}\right) \\
& =2 \int_{\mathbb{S}^{r}}\left(\sum_{k=1}^{m} \varepsilon_{k} I_{n-r-1, r}\left(\varrho_{k}\right)+I_{n-r-1, r}\left(\frac{\pi}{2 \sqrt{\lambda}}\right) N\left(\mathcal{C} \cap L_{r}^{r+1}\right)\right) \mathrm{d} u_{r},
\end{aligned}
$$

where $L_{r}^{r+1}$ denotes the $r$-plane perpendicular to the geodesic with direction $u_{r}$ from $O$ at a distance $\varrho=\frac{1}{2} \pi / \sqrt{\lambda}$.

Proof. The fact that $\mathcal{C}$ is a curve of class $C^{2}$ in $L_{r+1[0]}^{n}$ for a generic $(r+1)$ subspace $L_{r+1[0]}^{n}$ follows from Theorem A.1 of [8]. Consider first the case $\lambda \leqslant 0$. Then (4.2) may be written as

$$
\alpha\left(M^{n-r} \cap L_{r+1[0]}^{n}\right)=\int_{\mathbb{S}^{r}} \mathrm{~d} u_{r} \sum_{k=0}^{m-1} \int_{\varrho_{k}}^{\varrho_{k+1}} s_{\lambda}^{n-r-1}(\varrho) c_{\lambda}^{r}(\varrho) N\left(\mathcal{C} \cap L_{r}^{r+1}\right) \mathrm{d} \varrho,
$$

with the convention $\varrho_{0}=0$. Thus,

$$
\begin{aligned}
\alpha\left(M^{n-r} \cap L_{r+1[0]}^{n}\right) & =\int_{\mathbb{S}^{r}} \mathrm{~d} u_{r} \sum_{k=0}^{m-1} 2\left(I_{n-r-1, r}\left(\varrho_{k+1}\right)-I_{n-r-1, r}\left(\varrho_{k}\right)\right) \sum_{j=k+1}^{m} \varepsilon_{j} \\
& =2 \int_{\mathbb{S}^{r}}\left(\sum_{k=1}^{m} \varepsilon_{k} I_{n-r-1, r}\left(\varrho_{k}\right)-I_{n-r-1, r}(0) \sum_{k=1}^{m} \varepsilon_{k}\right) \mathrm{d} u_{r} .
\end{aligned}
$$

As we have set $I_{n-r-1, r}(0)=0$, the second term of the preceding integrand vanishes, and the proposed result is obtained.

For the case $\lambda>0$, the proof is similar. Thus,

$$
\begin{aligned}
& \alpha\left(M^{n-r} \cap L_{r+1[0]}^{n}\right) \\
& =\int_{\mathbb{S}^{r}} \mathrm{~d} u_{r} \sum_{k=0}^{m-1}\left(I_{n-r-1, r}\left(\varrho_{k+1}\right)-I_{n-r-1, r}\left(\varrho_{k}\right)\right)\left(2 \sum_{j=k+1}^{m} \varepsilon_{j}+N\left(\mathcal{C} \cap L_{r}^{r+1}\right)\right) \\
& \quad+\left(I_{n-r-1, r}(\pi / \sqrt{\lambda})-I_{n-r-1, r}\left(\varrho_{m}\right)\right) N\left(\mathcal{C} \cap L_{r}^{r+1}\right) .
\end{aligned}
$$

Therefore,

$$
\begin{aligned}
\alpha\left(M^{n-r} \cap\right. & \left.L_{r+1[0]}^{n}\right) \\
= & \int_{\mathbb{S}^{r}}\left(2 \sum_{k=1}^{m} \varepsilon_{k} I_{n-r-1, r}\left(\varrho_{k}\right)+I_{n-r-1, r}\left(\frac{\pi}{2 \sqrt{\lambda}}\right) N\left(\mathcal{C} \cap L_{r}^{r+1}\right)\right. \\
& \left.-I_{n-r-1, r}(0)\left(2 \sum_{k=1}^{m} \varepsilon_{k}+N\left(\mathcal{C} \cap L_{r}^{r+1}\right)\right)\right) \mathrm{d} u_{r},
\end{aligned}
$$

and because $I_{n-r-1, r}(0)=0$, the result follows. 
Remark 4.2. Equations (4.6) and (4.7) are pertinent to the representation of surface area (see the left-hand side of equation (3.6)). Hence, the integrands on the right-hand sides of equations (4.6) and (4.7) depend on the scanning direction $u_{r}$ in general. Note that the Euler characteristic is of no concern in this paper-we use Morse type concepts, but our route is different.

In order to see the relation between a critical point $p$ and the curvature, we consider a normal coordinate system of $L_{r+1[0]}^{n}$ at $p$. Let $\left\{U ; x_{1}, \ldots, x_{r+1}\right\}$ be a local chart described by the normal coordinate system centered at $p$; that is, there exist $U \subset \mathbb{R}^{r+1}, V \subset L_{r+1[0]}^{n}$, and a map $\varphi: U \rightarrow V$ which define the local chart.

Let $\alpha: I \subset \mathbb{R} \rightarrow L_{r+1[0]}^{n}$ be a parameterization of $\mathcal{C}$ with $\alpha(0)=p$, and set $\alpha:=\beta \circ \varphi$, with $\beta(0)=q \in \mathbb{R}^{r+1}$ and $\varphi(q)=p$.

Theorem 4.3. Given a point $p \in \mathcal{C}$ and a normal chart $\left\{U ; x_{i}\right\}$ at $p$, we have:

(1) $p$ is a critical point of $\left.h_{u_{r}}\right|_{\mathcal{C}}$ if and only if $\alpha^{\prime}(0) \perp \nu(p)$, where $\nu(p)$ is the unit vector perpendicular to the level set $L_{r}^{r+1} \subset L_{r+1[0]}^{n}$ at $p$.

(2) A critical point $p$ of $\left.h_{u_{r}}\right|_{\mathcal{C}}$ is nondegenerate if and only if $g\left(\nu(p), D^{2} \alpha(0)\right) \neq 0$, where $D$ is the operator of covariant differentiation with respect to $\alpha^{\prime}(t)$. Moreover, $p$ is a local maximum (minimum) if $g\left(\nu(p), D^{2} \alpha(0)\right)<0(>0)$.

P r o of. The point $p$ is a critical point of $\left.h_{u_{r}}\right|_{\mathcal{C}}$ if and only if $\left.\frac{\mathrm{d}}{\mathrm{d} t}\right|_{t=0}\left(h_{u_{r}} \circ \varphi \circ \beta\right)=0$. Using local coordinates yields

$$
\left.\frac{\mathrm{d}}{\mathrm{d} t}\right|_{t=0}\left(h_{u_{r}} \circ \varphi \circ \beta\right)=\left.\sum_{i=1}^{r+1} \frac{\partial\left(h_{u_{r}} \circ \varphi\right)}{\partial x_{i}}\right|_{q} x_{i}^{\prime}(0)=g\left(\operatorname{grad}\left(h_{u_{r}}\right)(p), \alpha^{\prime}(0)\right) .
$$

Then $p$ is a critical point of $\left.h_{u_{r}}\right|_{\mathcal{C}}$ if and only if $\operatorname{grad}\left(h_{u_{r}}\right)(p) \perp \alpha^{\prime}(0)$. Finally, since $\operatorname{grad}\left(h_{u_{r}}\right)(p) /\left\|\operatorname{grad}\left(h_{u_{r}}\right)(p)\right\|=\nu(p)$, the first part of the theorem follows.

To prove the second part we need to determine when $\left.\frac{\mathrm{d}^{2}}{\mathrm{~d} t^{2}}\right|_{t=0}\left(h_{u_{r}} \circ \varphi \circ \beta\right)$ vanishes. Using local coordinates yields

$$
\left.\frac{\mathrm{d}^{2}}{\mathrm{~d} t^{2}}\right|_{t=0}\left(h_{u_{r}} \circ \varphi \circ \beta\right)=\left.\sum_{i=1}^{r+1} \frac{\partial^{2}\left(h_{u_{r}} \circ \varphi\right)}{\partial x_{i} \partial x_{j}}\right|_{q} x_{i}^{\prime}(0) x_{j}^{\prime}(0)+\left.\sum_{i=1}^{r+1} \frac{\partial\left(h_{u_{r}} \circ \varphi\right)}{\partial x_{i}}\right|_{q} x_{i}^{\prime \prime}(0)
$$

The first term on the right-hand side of equation (4.13) is

$$
\begin{aligned}
\operatorname{hess}\left(h_{u_{r}}\right)\left(\alpha^{\prime}(0), \alpha^{\prime}(0)\right) & =g\left(\nabla_{\alpha^{\prime}(0)} \operatorname{grad}\left(h_{u_{r}}\right), \alpha^{\prime}(0)\right) \\
& =-|\varepsilon(p)| I I_{L_{r}}\left(\alpha^{\prime}(0)\right)=0
\end{aligned}
$$


where hess denotes the hessian and $I I_{L_{r}}$ is the second fundamental form of $L_{r}$ at $p$, which is zero, because $L_{r}$ is totally geodesic. As we have considered a normal coordinate system of $L_{r+1[0]}^{n}$ at $p$, the Christoffel symbols vanish; thus,

$$
D^{2} \alpha(0)=\left.\sum_{k=1}^{r+1} x_{k}^{\prime \prime}(0) \frac{\partial \varphi}{\partial x_{k}}\right|_{q}
$$

Further, the coefficients of the First Fundamental Form in normal coordinates are $E(p)=G(p)=1, F(p)=0$, whereby

$$
\begin{aligned}
\left.\frac{\mathrm{d}^{2}}{\mathrm{~d} t^{2}}\right|_{t=0} & \left(h_{u_{r}} \circ \varphi \circ \beta\right)=\left.\sum_{i=1}^{r+1} \frac{\partial\left(h_{u_{r}} \circ \varphi\right)}{\partial x_{i}}\right|_{q} x_{i}^{\prime \prime}(0) \\
& =g\left(\operatorname{grad}\left(h_{u_{r}}\right)(p), D^{2} \alpha(0)\right)=\left\|\operatorname{grad}\left(h_{u_{r}}\right)(p)\right\| g\left(\nu(p), D^{2} \alpha(0)\right)
\end{aligned}
$$

and the second part of the theorem follows.

$\mathrm{Remark}$ 4.4. If the curve $\alpha$ is parameterized by arc length, then from [9] we have that

$$
g\left(\nu(p), D^{2} \alpha(0)\right)=\kappa_{1} g\left(\nu(p), D^{2} \alpha(0) /\left\|D^{2} \alpha(0)\right\|\right)
$$

where $\kappa_{1}>0$ denotes the first curvature of $\alpha$.

Ex a m ple 4.5. Hypersurfaces in space forms.

Let $M^{n-1}$ be a hypersurface in $M_{\lambda}^{n}$. Theorem 4.1 yields

$$
\sigma_{n-1}\left(M^{n-1}\right)=\frac{2 O_{1}}{O_{n}} \int_{M^{n-1} \cap L_{2[0]}^{n} \neq \emptyset} \alpha\left(M^{n-1} \cap L_{2[0]}^{n}\right) \mathrm{d} L_{2[0]}^{n},
$$

where

$$
\alpha\left(M^{n-1} \cap L_{2[0]}^{n}\right)= \begin{cases}\int_{\mathbb{S}^{2} \cap L_{2[0]}^{n}} \sum_{k=1}^{m} \varepsilon_{k} \frac{s_{\lambda}^{n-1}\left(\varrho_{k}\right)}{n-1} \mathrm{~d} u, & \lambda<0, \\ \int_{\mathbb{S}^{2} \cap L_{2[0]}^{n}}\left(\sum_{k=1}^{m} \varepsilon_{k} \frac{s_{\lambda}^{n-1}\left(\varrho_{k}\right)}{n-1}+\frac{N\left(\mathcal{C} \cap L_{r}^{r+1}\right)}{n-1}\right) \mathrm{d} u, & \lambda>0 .\end{cases}
$$

From Theorem 4.3 it follows that $p$ is a critical point of $\left.h_{u_{r}}\right|_{\mathcal{C}}$ if and only if $\alpha^{\prime}(0)$ is perpendicular to the geodesic line $L_{1}^{2}$. Moreover, if $\alpha$ is parameterized by arc length, then

$$
g\left(\nu(p), D^{2} \alpha(0)\right)=\kappa_{g} g(\nu(p), N(0))
$$


where $N(0)$ is the unit vector normal to $\alpha^{\prime}(0)$ so that the orientation of $\left\{\alpha^{\prime}(s), N(s)\right\}$ agrees with the chosen orientation of $T_{p} L_{2[0]}^{n}$. Moreover, the sign of the geodesic curvature $\kappa_{g}$ of the curve at a point $p$ is adopted to be positive or negative according to whether the geodesic curvature vector lies to the right or the left of the curve within the geodesic surface $L_{2[0]}^{n}$. For $\lambda=0$ the geodesic curvature coincides with the signed curvature $\kappa$ of the plane curve.

\section{Application: Extension of the invariator method of Stereology TO ESTIMATE SURFACE AREA}

From equation (4.18) we obtain a simplified version of the Morse-type surface area estimator presented in equation (7) from [16]. We simply associate signed indexes to the critical points of the height function, without resorting to the concept of Euler characteristic. Let $Y$ be a compact set with smooth boundary $\partial Y$ in $\mathbb{R}^{3}$. From equation (4.18) we have that the surface area of $\partial Y$ can be expressed as follows:

$$
S(\partial Y)=\frac{1}{\pi} \int_{\mathbb{S}_{+}^{2}} \alpha\left(\partial Y \cap L_{2[0]}^{3}\right) \mathrm{d} L_{2[0]}^{3},
$$

where, by virtue of equation (4.19),

$$
\alpha\left(\partial Y \cap L_{2[0]}^{3}\right)=\int_{0}^{\pi} \mathrm{d} u \sum_{k=1}^{m} \varepsilon_{k} \varrho_{k}^{2} .
$$

For each axial direction $u \in[0, \pi)$ in the pivotal plane $L_{2[0]}^{3}$, the pivotal section is scanned entirely from top to bottom by a sweeping straight line parallel to the axis $O u$, in search of critical points. Above the axis $O u$, the value of the index $\varepsilon_{k}$ is +1 , or -1 , according to whether the $k$ th critical point is a local maximum, or a local minimum. Below the axis $O u$, it is convenient to imagine the pivotal section rotated by an angle of $180^{\circ}$, and then use the same criterion. As a consequence, the factor $1 /(n-1)$ (equal to $1 / 2$ in this case) on the right-hand side of equation (4.19) does not appear in (5.2). Because the integral of $\mathrm{d} L_{2[0]}^{3}$ over the unit hemisphere $\mathbb{S}_{+}^{2}$ is equal to $2 \pi$, the combination of the preceding two equations suggests the unbiased estimator of $S(\partial Y)$ from a single pivotal section, and from a single sweeping direction

$$
\widehat{S}(\partial Y)=2 \pi \sum_{k=1}^{m} \varepsilon_{k} \varrho_{k}^{2},
$$

which constitutes the aforementioned modification of equation (7) from [16]. The surface area estimator given by equation (3.2) from [5] corresponds to the case in 
which $Y$ is a convex set, whereby $m=2$. The latter estimator incorporated two mutually perpendicular sweeping directions. In this case, the summation on the right-hand side of equation (5.3) would be replaced with the mean of two summations. In [6], it was shown that two mutually perpendicular sweeping directions yield an accurate surface area estimation for ellipsoidal particles.

Example 5.1. Fig. 1(a) represents a pivotal section through a smooth particle $Y$, namely a section produced by an isotropically oriented plane $L_{2[0]}^{3}$ through a fixed pivotal point $O$ previously identifiable in the particle (e.g. a nucleolus of a neuron). The axis $O u$ has been conveniently oriented as horizontal, but it is supposed to be isotropically oriented about $O$.
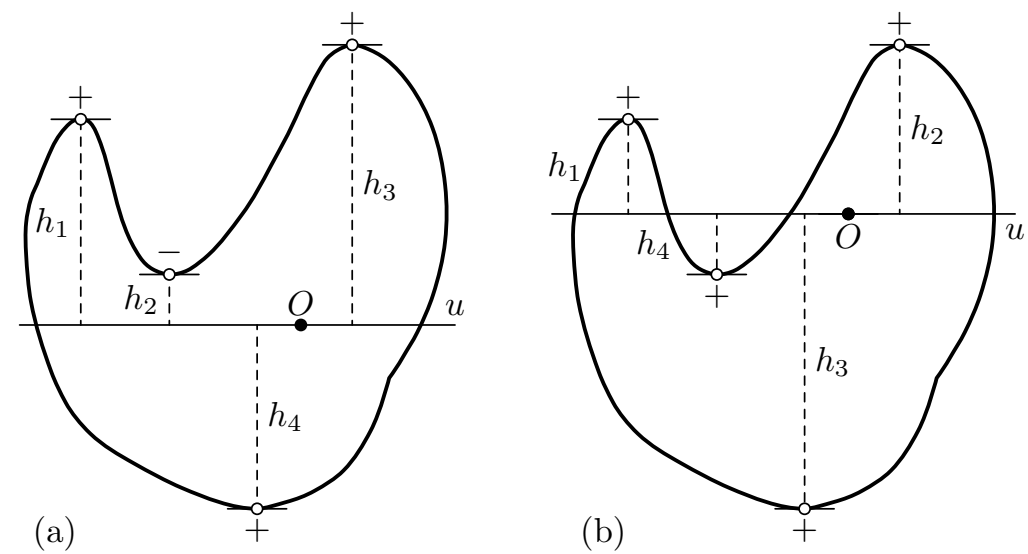

Figure 1. Illustration of the surface area estimator given by equation (5.3). See text, Example 5.1.

In Fig. 1(b), the section is the same, but for the sake of illustration, the pivotal point has a different location relative to the particle. A sweeping line moving parallelly to $\mathrm{Ou}$ from top to bottom determines four critical points in each case. In Fig. 1(a), the second critical point is a local minimum, whereby $\varepsilon_{2}=-1$. The remaining three critical points are local maxima, hence $\varepsilon_{1}=\varepsilon_{3}=\varepsilon_{4}=+1$. Thus, in this case,

$$
\widehat{S}(\partial X)=2 \pi\left(h_{1}^{2}-h_{2}^{2}+h_{3}^{2}+h_{4}^{2}\right)
$$

The distances to each critical point from the axis $O u$ have been denoted by $\left\{h_{k}\right\}$ instead of the $\left\{\varrho_{k}\right\}$ used in the rest of the paper, in order to match the notation with that adopted in [5], which evokes 'height' measure. In Fig. 1 (b), however, the four critical points are all local maxima, whereby, in this case,

$$
\widehat{S}(\partial X)=2 \pi\left(h_{1}^{2}+h_{2}^{2}+h_{3}^{2}+h_{4}^{2}\right)
$$


As suggested above, to identify the local maxima and minima below the axis $\mathrm{Ou}$, it is convenient to imagine the section as if turned upside down.

\section{References}

[1] J. Auneau, E. B. V. Jensen: Expressing intrinsic volumes as rotational integrals. Adv. Appl. Math. 45 (2010), 1-11.

[2] W. Blaschke: Integralgeometrie 1. Actualités Scientifiques et Industrielles 252, Hermann \& Cie., Paris, 1935. (In German.)

[3] E. Cartan: Le principe de dualité et certaines intégrales multiples de l'espace tangentiel et de l'espace réglé. Bull. Soc. Math. Fr. 24 (1896), 140-177. (In French.)

[4] M. W. Crofton: On the theory of local probability, applied to Straight Lines drawn at random in a plane; the methods used being also extended to the proof of certain new Theorems in the Integral Calculus. Philos. Trans. R. Soc. Lond. 158 (1868), 181-199. zbl

[5] L. M. Cruz-Orive: A new stereological principle for test lines in three-dimensional space. J. Microsc. 219 (2005), 18-28.

6] J. Dvořák, E. B. Jensen: On semiautomatic estimation of surface area. J. Microsc. 250 (2013), 142-57.

[7] X. Gual-Arnau, L. M. Cruz-Orive: A new expression for the density of totally geodesic submanifolds in space forms, with stereological applications. Differ. Geom. Appl. 27 (2009), 124-128.

[8] X. Gual-Arnau, L. M. Cruz-Orive, J. J. Nuño-Ballesteros: A new rotational integral formula for intrinsic volumes in space forms. Adv. Appl. Math. 44 (2010), 298-308.

[9] E. Gutkin: Curvatures, volumes and norms of derivatives for curves in Riemannian manifolds. J. Geom. Phys. 61 (2011), 2147-2161.

[10] M.W. Hirsch: Differential Topology. Corrected reprint of the 1976 original. Graduate Texts in Mathematics 33, Springer, New York, 1994.

[11] B. Petkantschin: Integralgeometrie 6. Zusammenhänge zwischen den Dichten der linearen Unterräume im n-dimensionalen Raum. Abh. Math. Semin. Hamb. Univ. 11 (1936), 249-310. (In German.)

[12] D.-l. Ren: Topics in Integral Geometry. Series in Pure Mathematics 19, World Scientific, Singapore, 1994.

13] L. A. Santaló: Integral Geometry and Geometric Probability. Cambridge Mathematical Library, Cambridge University Press, Cambridge, 2004.

[14] R. Schneider, W. Weil: Stochastic and Integral Geometry. Probability and Its Applications, Springer, Berlin, 2008.

[15] Ó. Thórisdóttir, M. Kiderlen: The invariator principle in convex geometry. Adv. Appl. Math. 58 (2014), 63-87.

[16] Ó. Thórisdóttir, A.H.Rafati, M. Kiderlen: Estimating the surface area of nonconvex particles from central planar sections. J. Micrsoc. 255 (2014), 49-64.

Authors' addresses: Ximo Gual-Arnau, Departament de Matemàtiques-INIT, Universitat Jaume I, E-12071-Castelló de la Plana, Spain, e-mail: gual@uji.es; Luis M. Cruz-Orive, Departamento de Matemáticas, Estadística y Computación, Universidad de Cantabria, E-39005-Santander, Spain, e-mail: luis.cruz@unican.es. 